\title{
Determination of the temperatures of selected ISO flux calibration stars using the Infrared Flux Method ${ }^{\star}$
}

\author{
D.E. Blackwell and A.E. Lynas-Gray \\ Astrophysics, Nuclear Physics Laboratory, Keble Road, Oxford OX1 3RH, UK
}

Received April 1; accepted October 6, 1997

\begin{abstract}
Effective temperatures for 420 stars with spectral types between A0 and K3, and luminosity classes between II and V, selected for a flux calibration of the Infrared Space Observatory, ISO, have been determined using the Infrared Flux Method (IRFM). The determinations are based on narrow and wide band photometric data obtained for this purpose, and take into account previously published narrow-band measures of temperature. Regression coefficients are given for relations between the determined temperatures and the photometric parameters $(B 2-V 1),(b-y)$ and $(B-V)$, corrected for interstellar extinction through use of Hipparcos parallaxes. A correction for the effect of metallicity on the determination of integrated flux is proposed. The importance of a knowledge of metallicity in the representation of derived temperatures for Class V, IV and III stars by empirical functions is discussed and formulae given. An estimate is given for the probable error of each temperature determination.
\end{abstract}

Key words: stars: fundamental parameters temperatures

\section{Introduction}

The purpose of the investigation is the determination of accurate (one per cent) temperatures for selected stars for use in a flux calibration for ISO, as described by van der Bliek et al. (1992) using the Infrared Flux Method. The method and its applications have been discussed in detail by Blackwell et al. (1980, 1990, 1991), Blackwell \& Lynas-Gray (1994), Saxner \& Hammarbäck (1985), Bell \& Gustafsson (1989), Smalley (1993), Glushneva et al. (1993) and Alonso et al. (1995). Because of known limitations of the IRFM, the range of stars is restricted to

\footnotetext{
Send offprint requests to: D.E. Blackwell

* Based on data from the ESA Hipparcos Astrometry Satellite.
}

those with temperatures between $10000 \mathrm{~K}$ and $4200 \mathrm{~K}$, and surface gravities between $\log g=4.5$ and 1.0.

The method is based on the measurement of two stellar quantities: the absolute monochromatic continuum flux density, $F(\lambda)$, at a chosen wavelength $\lambda$ in the near infrared, and the absolute integrated flux $F$. The ratio of these two quantities is related to the stellar temperature $T$ through the equation

$R=F / F(\lambda)=\sigma T^{4} / \psi(T, g, \lambda, A)$

where $\psi(T, g, \lambda, A)$ gives the monochromatic flux from the star as a function of effective temperature, the surface gravity, the wavelength at which the monochromatic flux is determined, and the atomic abundances. The method is applied by calculating the right-hand side of the equation as a function of temperature for a chosen value of $\lambda$ on the Rayleigh-Jeans tail, and for selected values of $\log g$ and $A$, using a range of model stellar atmospheres. This procedure gives a calibration relating temperatures to measured values of $R$. Later calibrations, for example those of Blackwell \& Lynas-Gray (1994) have been made using more recent models by Kurucz $(1991,1992)$. Other calibrations have been published by Alonso et al. (1996a) for dwarf and subdwarf stars, and by Mégessier (1994). The accuracy of flux calculations using model atmospheres has been considered by van der Bliek et al. (1996), whilst Mégessier (1994) has assessed the influence of the model atmosphere on derivations of temperature.

The dependence of the IRFM on stellar metallicity has been discussed by Mégessier (1994) and by Smalley (1993). However, an accurate correction for metallicity cannot always be made for the present program stars because of a lack of precise measurements of $[\mathrm{Fe} / \mathrm{H}]$. In addition, even the solar iron abundance is now uncertain to the extent of 0.12 dex following the work of, for example, Holweger et al. (1991, 1995) and Blackwell et al. (1995). Most of the basic $[\mathrm{Fe} / \mathrm{H}]$ values adopted for the stars in this paper have been taken from the survey of measurements by Cayrel de Strobel et al. (1992), supplemented by those given by Edvardsson et al. (1993). Where there are no direct measures, Schuster \& Nissen (1989) and Carney (1979) have 
suggested methods of deriving $[\mathrm{Fe} / \mathrm{H}]$ depending on stellar photometry, but the results are probably uncertain to 0.6 dex. In these circumstances, we have assumed a mean value of $[\mathrm{Fe} / \mathrm{H}]=-0.1$ dex for stars for which there are no direct measures of $[\mathrm{Fe} / \mathrm{H}]$, a value which corresponds to the mean measured value of $[\mathrm{Fe} / \mathrm{H}]$ for all of the stars in this program. For the majority of stars, these procedures should give results with a mean temperature error of less than 0.5 per cent through uncertainty in metallicity.

The IRFM requires values of interstellar extinction so that corrections can be made to observed infrared and integrated fluxes. Blackwell \& Lynas-Gray (1994) give a table showing the effect of interstellar extinction on the determination of temperature. In the present work, individual extinction values have been obtained using parallaxes measured by the Hipparcos satellite (ESA 1997). The Hipparcos parallaxes for the more distant stars have been of the utmost importance, for they have enabled the the IRFM to be greatly extended in its range of application. Following previous work we assume an average interstellar extinction of $A_{V}=0.8 \mathrm{mag} / \mathrm{kpc}$, with Allen's (1955) wavelength dependence, but also consider the results of the complex mapping of extinction made by Arenou et al. (1992).

\section{Application of the IRFM to ISO calibration stars}

\subsection{Use of published narrow band infrared magnitudes}

Two studies (I and II) of stellar temperatures and angular diameters determined using the IRFM with narrow band filters have recently been published (Blackwell et al. 1990) and Blackwell \& Lynas-Gray (1994). The second of these two studies (II) presents potentially more accurate results through the use of new Kurucz LTE line-blanketed model atmospheres instead of the MARCS code of Gustafsson et al. (1975), which was used in study (I), for calibration of the method. However, the results given by these two studies differ only very slightly: the ratio of the temperatures $T(\mathrm{II}) / T(\mathrm{I})=0.9995$, and the standard deviation of the ratio $\sigma=0.0078$. In view of the closeness of the results of these two studies, we incorporate them in our analysis of new data.

\subsection{The database of magnitudes in the visible region}

In the subsequent sections, values for the Geneva photometric quantities $(B-V)$ and $(B 2-V 1)$ have been taken from Rufener (1988). Those for $(b-y)$ magnitudes have been taken chiefly from Heck (1977), Olsen (1977, 1983, 1993).

\subsection{The database of infrared magnitudes}

The temperatures discussed in this paper are based on stellar photometry in the $J, H, K$ and $L$ regions, obtained by van der Bliek et al. (1996), hereafter ESO data, and by Dr. P. Hammersley (to be published) using the Carlos Sánchez telescope at the Observatorio del Teide, hereafter TCS data. Because of the limitations of the IRFM, we have restricted the range of stars to those with temperatures between $4200 \mathrm{~K}$ and $10000 \mathrm{~K}$ and surface gravities between $\log g=1.0$ and 4.5. The IRFM is unsuitable for hotter stars because they have a large and uncertain ultraviolet contribution to the integrated flux, and in addition, the interstellar extinction is less reliable in this region. Stars cooler than $4200 \mathrm{~K}$ have not been considered because infrared fluxes at longer $(>3.8 \mu \mathrm{m})$ wavelengths would be needed.

\subsection{Absolute flux determinations}

The filter profiles from the sources listed in Sects. 2.1 and 2.3 ranged from narrow to wide, so that several different calibrations were needed. All data were reduced by integration of the stellar flux distribution and atmospheric transmission profiles over the filter profiles to give a flux at a particular chosen wavelength. These fluxes were converted to absolute fluxes using Vega fluxes determined by Walker \& Cohen (1992), based on the very careful and thorough work by Cohen et al. (1992). When all of the data had been used for the determination of temperature, it became clear that determinations using the wide $J$ filters were less reliable because of the complexity of the filter profiles and the complication caused by some stellar and telluric absorption lines in that region. Accordingly, some of the $J$ filter temperature determinations have either been given lower weight or have been rejected.

Because of the difficulty of determining accurate monochromatic fluxes from the more complex filter profiles, we have tried, for checking purposes only, a completely different method based on the previously published narrow band determinations. There are stars that are common between this original study and the ISO calibration list. Believing that the results of the original study are of good accuracy because of the unique suitability of the narrow band filters, we can use these stars to determine filter factors that should be used in the ISO study - that is, for each star in common between the two lists, we can derive individual filter factors that equalise both narrow band pairs of temperatures. An average is then taken of all the factors found in this way to apply to the new data. Such factors will constrain the ISO temperatures to fit the scale of the original narrow band IRFM temperatures. This method has proved very useful for assessment purposes only, and its results confirm the more direct integrations used for the various wider band ESO and TCS data. Temperatures found using individual filters are consistent to within about one per cent. Final temperatures have been derived by averaging those found with available filters, excluding $J$ filters whenever practicable. 
Table 1. Total Fluxes and derived temperatures for ISO Stars. Column S lists the sources of infrared data: A denotes Telescopio Carlos Sanchez (TCS) data, B denotes ESO data. PE lists the estimated probable errors of derived temperatures expressed as a percentage

\begin{tabular}{|c|c|c|c|c|c|c|c|c|c|c|c|c|c|c|}
\hline HD & FLUX & TEMP & $\mathrm{S}$ & $\overline{\mathrm{PE}}$ & HD & FLUX & TEMP & $\mathrm{S}$ & $\mathrm{PE}$ & HD & FLUX & TEMP & $\mathrm{S}$ & $\mathrm{PE}$ \\
\hline 123 & $1.140 \mathrm{E}-10$ & 5601 & $\mathrm{~A}$ & 0.9 & 18411 & $3.636 \mathrm{E}-10$ & 8558 & $\mathrm{~A}$ & 0.9 & 45320 & $1.247 \mathrm{E}-10$ & 8785 & $\mathrm{~A}$ & 0.7 \\
\hline 693 & $2.883 \mathrm{E}-10$ & 6143 & B & 0.8 & 18702 & $1.759 \mathrm{E}-11$ & 5268 & A & 0.9 & 45701 & $7.286 \mathrm{E}-11$ & 5865 & B & 1.0 \\
\hline 787 & $4.438 \mathrm{E}-10$ & 4181 & B & 1.2 & 18978 & $6.080 \mathrm{E}-10$ & 7855 & B & 0.9 & 46251 & $7.392 \mathrm{E}-11$ & 9045 & A & 0.6 \\
\hline 1368 & $7.820 \mathrm{E}-12$ & 5887 & A & 0.9 & 19066 & $1.038 \mathrm{E}-10$ & 4900 & A & 0.9 & 47174 & $5.186 \mathrm{E}-10$ & 4394 & A & 1.0 \\
\hline 1581 & $5.468 \mathrm{E}-10$ & 5867 & B & 0.5 & 19373 & $6.372 \mathrm{E}-10$ & 5981 & A & 0.6 & 47205 & $9.172 \mathrm{E}-10$ & 4773 & B & 0.9 \\
\hline 1835 & $7.590 \mathrm{E}-11$ & 5713 & B & 2.0 & 19476 & $9.890 \mathrm{E}-10$ & 5018 & A & 1.2 & 47442 & $7.190 \mathrm{E}-10$ & 4633 & A & 0.9 \\
\hline 2151 & $2.019 \mathrm{E}-09$ & 5710 & B & 0.5 & 19656 & $5.296 \mathrm{E}-10$ & 4714 & A & 0.9 & 49293 & $5.957 \mathrm{E}-10$ & 4653 & A & 0.9 \\
\hline 2262 & $6.688 \mathrm{E}-10$ & 7882 & B & 0.8 & 20630 & $3.217 \mathrm{E}-10$ & 5713 & B & 1.0 & 49933 & $1.279 \mathrm{E}-10$ & 6484 & A & 0.7 \\
\hline 2811 & $3.013 \mathrm{E}-11$ & 8317 & B & 0.9 & 20766 & $1.703 \mathrm{E}-10$ & 5770 & B & 1.5 & 50241 & $1.222 \mathrm{E}-09$ & 7372 & B & 0.9 \\
\hline 3443 & $1.743 \mathrm{E}-10$ & 5240 & A & 0.7 & 20794 & $5.720 \mathrm{E}-10$ & 5420 & B & 0.7 & 50522 & $5.687 \mathrm{E}-10$ & 5077 & A & 0.9 \\
\hline 3627 & $2.134 \mathrm{E}-09$ & 4335 & A & 0.7 & 21120 & $1.117 \mathrm{E}-09$ & 5031 & A & 0.9 & 53705 & $1.659 \mathrm{E}-10$ & 5753 & B & 0.9 \\
\hline 3628 & $3.255 \mathrm{E}-11$ & 5673 & A & 0.9 & 21543 & $1.443 \mathrm{E}-11$ & 5650 & A & 0.9 & 53706 & $5.591 \mathrm{E}-11$ & 5325 & B & 1.0 \\
\hline 3919 & $5.415 \mathrm{E}-10$ & 5100 & B & 1.2 & 22484 & $5.125 \mathrm{E}-10$ & 5944 & B & 0.5 & 53811 & $2.804 \mathrm{E}-10$ & 8203 & B & 0.9 \\
\hline 4128 & $5.168 \mathrm{E}-10$ & 4853 & B & 0.9 & 22879 & $5.766 \mathrm{E}-11$ & 5762 & A & 0.7 & 54810 & $3.906 \mathrm{E}-10$ & 4758 & B & 0.9 \\
\hline 5448 & $7.253 \mathrm{E}-10$ & 7981 & A & 0.9 & 23763 & $4.948 \mathrm{E}-11$ & 8441 & A & 0.9 & 56169 & $2.640 \mathrm{E}-10$ & 8216 & A & 0.5 \\
\hline 5516 & $5.588 \mathrm{E}-10$ & 4970 & A & 0.9 & 24160 & $6.912 \mathrm{E}-10$ & 5089 & B & 0.9 & 56405 & $1.774 \mathrm{E}-10$ & 8721 & B & 1.0 \\
\hline 6582 & $2.504 \mathrm{E}-10$ & 5292 & A & 0.5 & 24341 & $2.107 \mathrm{E}-11$ & 5462 & A & 0.9 & 56513 & $1.714 \mathrm{E}-11$ & 5642 & A & 0.9 \\
\hline 6595 & $1.497 \mathrm{E}-09$ & 5116 & B & 0.9 & 24849 & $3.848 \mathrm{E}-12$ & 6483 & B & 1.2 & 58207 & $1.073 \mathrm{E}-09$ & 4766 & A & 0.8 \\
\hline 6619 & $7.004 \mathrm{E}-11$ & 8750 & B & 0.9 & 25329 & $1.377 \mathrm{E}-11$ & 4834 & A & 0.5 & 58946 & $5.282 \mathrm{E}-10$ & 6821 & A & 1.2 \\
\hline 6660 & $1.763 \mathrm{E}-11$ & 4547 & A & 0.9 & 25532 & $1.800 \mathrm{E}-11$ & 5333 & A & 0.9 & 59294 & $6.307 \mathrm{E}-10$ & 4482 & A & 0.9 \\
\hline 6755 & $2.737 \mathrm{E}-11$ & 5127 & A & 2.0 & 25604 & $6.439 \mathrm{E}-10$ & 4799 & A & 1.2 & 59967 & $6.020 \mathrm{E}-11$ & 5732 & B & 0.9 \\
\hline 6961 & $4.747 \mathrm{E}-10$ & 7998 & A & 0.9 & 26612 & $2.760 \mathrm{E}-10$ & 6944 & B & 0.9 & 59984 & $1.177 \mathrm{E}-10$ & 5896 & A & 0.6 \\
\hline 7424 & $2.732 \mathrm{E}-12$ & 5648 & B & 1.0 & 26756 & $1.244 \mathrm{E}-11$ & 5617 & A & 0.9 & 60298 & $3.115 \mathrm{E}-11$ & 5780 & A & 0.9 \\
\hline 8799 & $2.938 \mathrm{E}-10$ & 6520 & A & 1.2 & 26965 & $5.353 \mathrm{E}-10$ & 5161 & A & 0.9 & 60778 & $7.310 \mathrm{E}-12$ & 8370 & B & 0.7 \\
\hline 9138 & $6.421 \mathrm{E}-10$ & 4063 & B & 0.7 & 26967 & 1.091E-09 & 4644 & B & 0.9 & 61110 & $2.832 \mathrm{E}-10$ & 6535 & A & 0.6 \\
\hline 9362 & $9.174 \mathrm{E}-10$ & 4840 & B & 0.9 & 27697 & $1.014 \mathrm{E}-09$ & 4928 & A & 0.9 & 61935 & $9.324 \mathrm{E}-10$ & 4814 & B & 0.9 \\
\hline 9562 & $1.366 \mathrm{E}-10$ & 5791 & B & 0.9 & 27741 & $1.392 \mathrm{E}-11$ & 5765 & A & 0.9 & 62044 & $7.555 \mathrm{E}-10$ & 4585 & A & 0.9 \\
\hline 9826 & $5.958 \mathrm{E}-10$ & 6136 & A & 0.5 & 27819 & $3.103 \mathrm{E}-10$ & 8037 & A & 0.6 & 62509 & $1.047 \mathrm{E}-08$ & 4837 & A & 0.6 \\
\hline 9927 & $1.610 \mathrm{E}-09$ & 4360 & A & 0.9 & 28100 & $4.488 \mathrm{E}-10$ & 4882 & A & 0.9 & 64090 & $1.388 \mathrm{E}-11$ & 5313 & A & 0.6 \\
\hline 10126 & $2.356 \mathrm{E}-11$ & 5538 & A & 0.9 & 28305 & $1.280 \mathrm{E}-09$ & 4911 & A & 0.7 & 64096 & $2.354 \mathrm{E}-10$ & 5771 & A & 0.7 \\
\hline 10476 & $2.442 \mathrm{E}-10$ & 5223 & A & 1.2 & 29291 & $9.828 \mathrm{E}-10$ & 5015 & B & 0.7 & 64606 & $3.279 \mathrm{E}-11$ & 5109 & A & 0.5 \\
\hline 10697 & $8.748 \mathrm{E}-11$ & 5612 & A & 0.9 & 29310 & $2.738 \mathrm{E}-11$ & 5781 & A & 0.9 & 65430 & $2.605 \mathrm{E}-11$ & 5127 & A & 0.9 \\
\hline 10700 & $1.162 \mathrm{E}-09$ & 5337 & B & 0.9 & 29388 & $5.082 \mathrm{E}-10$ & 8282 & A & 0.9 & 65583 & $4.754 \mathrm{E}-11$ & 5233 & A & 0.5 \\
\hline 10800 & $1.215 \mathrm{E}-10$ & 5835 & B & 0.9 & 30562 & $1.337 \mathrm{E}-10$ & 5843 & A & 0.7 & 69897 & $2.320 \mathrm{E}-10$ & 6217 & A & 0.5 \\
\hline 11353 & $1.215 \mathrm{E}-09$ & 4685 & B & 0.9 & 30652 & $1.378 \mathrm{E}-09$ & 6380 & B & 0.6 & 71155 & $8.670 \mathrm{E}-10$ & 9663 & B & 0.9 \\
\hline 11443 & $1.096 \mathrm{E}-09$ & 6321 & A & 0.9 & 31966 & $5.879 \mathrm{E}-11$ & 5696 & A & 0.8 & 73108 & $5.947 \mathrm{E}-10$ & 4524 & A & 0.7 \\
\hline 11946 & $2.426 \mathrm{E}-10$ & 9681 & A & 0.9 & 33276 & $3.127 \mathrm{E}-10$ & 7249 & A & 1.2 & 73524 & $6.492 \mathrm{E}-11$ & 5860 & B & 0.9 \\
\hline 12311 & $1.797 \mathrm{E}-09$ & 7165 & B & 0.9 & 34411 & $3.520 \mathrm{E}-10$ & 5848 & A & 0.5 & 73665 & $9.254 \mathrm{E}-11$ & 5022 & A & 0.9 \\
\hline 13041 & $3.152 \mathrm{E}-10$ & 8242 & A & 0.9 & 36283 & $1.065 \mathrm{E}-11$ & 5616 & A & 1.0 & 74918 & $6.118 \mathrm{E}-10$ & 5105 & B & 0.9 \\
\hline 13201 & $7.113 \mathrm{E}-11$ & 6357 & A & 0.5 & 37160 & $8.162 \mathrm{E}-10$ & 4701 & A & 0.7 & 76932 & $1.282 \mathrm{E}-10$ & 5719 & A & 0.6 \\
\hline 13403 & $4.530 \mathrm{E}-11$ & 5577 & A & 0.5 & 37394 & $9.871 \mathrm{E}-11$ & 5179 & A & 0.5 & 78004 & $1.280 \mathrm{E}-09$ & 4588 & B & 0.9 \\
\hline 13783 & $1.375 \mathrm{E}-11$ & 5527 & A & 0.7 & 37507 & $3.131 \mathrm{E}-10$ & 8102 & A & 0.9 & 79917 & $3.988 \mathrm{E}-10$ & 4675 & B & 0.9 \\
\hline 13836 & $1.650 \mathrm{E}-11$ & 5561 & A & 0.9 & 38393 & $9.446 \mathrm{E}-10$ & 6210 & B & 0.5 & 81809 & $1.988 \mathrm{E}-10$ & 5619 & A & 0.6 \\
\hline 13974 & $3.158 \mathrm{E}-10$ & 5594 & A & 0.6 & 38656 & $5.131 \mathrm{E}-10$ & 4898 & A & 0.9 & 84937 & $1.273 \mathrm{E}-11$ & 6202 & A & 1.2 \\
\hline 14770 & $2.812 \mathrm{E}-10$ & 4951 & A & 0.9 & 38858 & $1.129 \mathrm{E}-10$ & 5669 & B & 0.9 & 85444 & $7.313 \mathrm{E}-10$ & 5085 & B & 0.9 \\
\hline 15008 & $6.533 \mathrm{E}-10$ & 8936 & B & 0.9 & 39060 & $7.258 \mathrm{E}-10$ & 8045 & B & 1.2 & 87140 & $8.789 \mathrm{E}-12$ & 5172 & $\mathrm{~A}$ & 2.0 \\
\hline 15189 & $5.256 \mathrm{E}-12$ & 6450 & B & 1.5 & 40035 & $1.099 \mathrm{E}-09$ & 4764 & A & 0.9 & 88371 & $1.229 \mathrm{E}-11$ & 5641 & A & 0.9 \\
\hline 15798 & $3.305 \mathrm{E}-10$ & 6331 & A & 0.9 & 40136 & $8.185 \mathrm{E}-10$ & 7006 & B & 0.7 & 88742 & $7.552 \mathrm{E}-11$ & 5861 & B & 0.8 \\
\hline 16031 & $3.453 \mathrm{E}-12$ & 6094 & B & 0.7 & 40832 & $8.375 \mathrm{E}-11$ & 6489 & A & 0.9 & 89010 & $1.140 \mathrm{E}-10$ & 5686 & A & 0.5 \\
\hline 16160 & $1.725 \mathrm{E}-10$ & 4759 & A & 0.5 & 43039 & $6.709 \mathrm{E}-10$ & 4737 & A & 0.8 & 89125 & $1.249 \mathrm{E}-10$ & 6028 & A & 0.6 \\
\hline 16397 & $3.147 \mathrm{E}-11$ & 5762 & A & 0.9 & 43147 & $7.229 \mathrm{E}-12$ & 5158 & A & 0.9 & 90508 & $7.320 \mathrm{E}-11$ & 5721 & A & 0.6 \\
\hline 16815 & $8.202 \mathrm{E}-10$ & 4766 & B & 0.5 & 44007 & $2.300 \mathrm{E}-11$ & 4910 & A & 0.9 & 91324 & $2.930 \mathrm{E}-10$ & 6124 & B & 0.7 \\
\hline 17652 & $5.762 \mathrm{E}-10$ & 4844 & B & 0.9 & 44420 & $2.533 \mathrm{E}-11$ & 5804 & A & 1.0 & 92764 & $7.154 \mathrm{E}-12$ & 8019 & A & 0.9 \\
\hline 17674 & $2.586 \mathrm{E}-11$ & 5875 & A & 0.9 & 44594 & $6.263 \mathrm{E}-11$ & 5741 & B & 0.7 & 93702 & $2.140 \mathrm{E}-10$ & 8878 & A & 0.9 \\
\hline 17824 & $3.968 \mathrm{E}-10$ & 5063 & B & 0.9 & 45184 & $7.791 \mathrm{E}-11$ & 5790 & B & 0.9 & 93813 & $2.510 \mathrm{E}-09$ & 4431 & B & 0.9 \\
\hline
\end{tabular}


Table 1. continued

\begin{tabular}{|c|c|c|c|c|c|c|c|c|c|c|c|c|c|c|}
\hline HD & FLUX & TEMP & $\mathrm{S}$ & $\overline{\mathrm{PE}}$ & HD & FLUX & TEMP & $\mathrm{S}$ & $\overline{\mathrm{PE}}$ & $\mathrm{HD}$ & FLUX & TEMP & $\mathrm{S}$ & $\mathrm{PE}$ \\
\hline 94028 & $1.399 \mathrm{E}-11$ & 5998 & $\bar{A}$ & 0.8 & 120136 & $4.009 \mathrm{E}-10$ & 6424 & $\bar{A}$ & 0.9 & 144284 & $6.497 \mathrm{E}-10$ & 6138 & $\bar{A}$ & 0.5 \\
\hline 94510 & $1.012 \mathrm{E}-09$ & 4938 & B & 0.9 & 120452 & $3.685 \mathrm{E}-10$ & 4740 & A & 0.9 & 144579 & $6.505 \mathrm{E}-11$ & 5309 & A & 0.9 \\
\hline 94890 & $5.033 \mathrm{E}-10$ & 4837 & B & 0.9 & 120787 & $1.459 \mathrm{E}-10$ & 4936 & A & 0.9 & 146686 & $8.798 \mathrm{E}-10$ & 4766 & B & 0.9 \\
\hline 95370 & $4.608 \mathrm{E}-10$ & 8217 & B & 0.9 & 121131 & $1.414 \mathrm{E}-11$ & 5131 & A & 0.9 & 146791 & $1.694 \mathrm{E}-09$ & 4882 & A & 0.9 \\
\hline 95934 & $1.009 \mathrm{E}-10$ & 7966 & A & 0.9 & 121474 & $4.867 \mathrm{E}-10$ & 4694 & B & 0.9 & 147513 & $1.887 \mathrm{E}-10$ & 5679 & B & 0.9 \\
\hline 96566 & $4.786 \mathrm{E}-10$ & 4913 & B & 0.9 & 123977 & $9.752 \mathrm{E}-11$ & 4845 & A & 0.9 & 147547 & 7.687E-10 & 7125 & A & 0.5 \\
\hline 96574 & $3.189 \mathrm{E}-11$ & 6115 & A & 1.0 & 124850 & $6.132 \mathrm{E}-10$ & 6168 & A & 0.9 & 148387 & 2.494E-09 & 5007 & A & 0.5 \\
\hline 96833 & $2.359 \mathrm{E}-09$ & 4650 & A & 0.9 & 125161 & $3.190 \mathrm{E}-10$ & 7792 & A & 0.9 & 148786 & $6.159 \mathrm{E}-10$ & 5106 & B & 0.9 \\
\hline 97503 & $1.452 \mathrm{E}-11$ & 4351 & A & 0.9 & 125454 & $3.193 \mathrm{E}-10$ & 4845 & A & 0.9 & 148816 & $3.352 \mathrm{E}-11$ & 5833 & A & 0.9 \\
\hline 97916 & $5.699 \mathrm{E}-12$ & 6409 & A & 0.6 & 126053 & $8.609 \mathrm{E}-11$ & 5635 & B & 0.9 & 148856 & $2.406 \mathrm{E}-09$ & 4983 & A & 0.9 \\
\hline 98562 & 8.687E-12 & 5883 & A & 1.0 & 126681 & $5.457 \mathrm{E}-12$ & 5558 & A & 1.0 & 149414 & $4.688 \mathrm{E}-13$ & 5048 & A & 0.8 \\
\hline 99747 & $1.184 \mathrm{E}-10$ & 6547 & A & 1.0 & 127243 & $1.946 \mathrm{E}-10$ & 5020 & A & 0.9 & 150997 & $1.274 \mathrm{E}-09$ & 4918 & A & 0.7 \\
\hline 100407 & $1.228 \mathrm{E}-09$ & 5058 & B & 0.9 & 127665 & 1.663E-09 & 4220 & A & 0.9 & 151680 & $4.834 \mathrm{E}-09$ & 4489 & B & 0.9 \\
\hline 101206 & $1.996 \mathrm{E}-11$ & 4576 & A & 0.9 & 128167 & $4.087 \mathrm{E}-10$ & 6737 & A & 0.5 & 152156 & $4.413 \mathrm{E}-12$ & 5017 & A & 0.8 \\
\hline 101606 & $1.323 \mathrm{E}-10$ & 6193 & A & 0.5 & 128429 & 8.659E-11 & 6336 & A & 0.5 & 152306 & $5.255 \mathrm{E}-11$ & 4976 & A & 0.9 \\
\hline 102249 & $9.013 \mathrm{E}-10$ & 7908 & B & 0.9 & 128959 & $6.203 \mathrm{E}-12$ & 5622 & A & 0.9 & 152311 & $1.273 \mathrm{E}-10$ & 5588 & A & 0.8 \\
\hline 102365 & $3.022 \mathrm{E}-10$ & 5538 & B & 0.5 & 129502 & $7.164 \mathrm{E}-10$ & 6642 & B & 0.9 & 153312 & $1.143 \mathrm{E}-10$ & 4752 & A & 0.9 \\
\hline 102634 & $9.137 \mathrm{E}-11$ & 6317 & A & 0.6 & 129685 & $3.155 \mathrm{E}-10$ & 9089 & B & 0.9 & 154363 & $3.809 \mathrm{E}-11$ & 4340 & A & 0.9 \\
\hline 102870 & $9.590 \mathrm{E}-10$ & 6124 & A & 0.5 & 129858 & $1.508 \mathrm{E}-10$ & 8968 & B & 0.9 & 155125 & 3.341E-09 & 8690 & A & 0.9 \\
\hline 103095 & $8.340 \mathrm{E}-11$ & 5054 & A & 0.5 & 130163 & $6.180 \mathrm{E}-11$ & 9720 & B & 0.5 & 156026 & $1.288 \mathrm{E}-10$ & 4377 & A & 0.6 \\
\hline 103912 & $1.608 \mathrm{E}-11$ & 5048 & A & 0.9 & 130871 & $8.062 \mathrm{E}-12$ & 4785 & A & 0.9 & 156164 & $1.478 \mathrm{E}-09$ & 8364 & A & 1.2 \\
\hline 104076 & $1.425 \mathrm{E}-10$ & 5884 & A & 0.9 & 130948 & $1.205 \mathrm{E}-10$ & 5995 & A & 0.6 & 156277 & $5.387 \mathrm{E}-10$ & 4499 & B & 0.9 \\
\hline 104381 & $4.844 \mathrm{E}-11$ & 8606 & A & 0.9 & 131977 & $2.011 \mathrm{E}-10$ & 4609 & A & 0.9 & 157089 & $4.574 \mathrm{E}-11$ & 5732 & A & 0.8 \\
\hline 104979 & $7.408 \mathrm{E}-10$ & 4903 & A & 0.9 & 132052 & 4.007E-10 & 6980 & A & 0.9 & 157740 & $1.460 \mathrm{E}-10$ & 8758 & A & 1.0 \\
\hline 105452 & $6.172 \mathrm{E}-10$ & 6875 & B & 0.9 & 132142 & $2.417 \mathrm{E}-11$ & 5143 & A & 0.9 & 157950 & $3.813 \mathrm{E}-10$ & 6588 & A & 1.0 \\
\hline 105755 & $1.038 \mathrm{E}-11$ & 5784 & A & 0.7 & 132475 & $1.109 \mathrm{E}-11$ & 5677 & A & 0.9 & 158226 & $1.128 \mathrm{E}-11$ & 5823 & A & 1.0 \\
\hline 106516 & $9.432 \mathrm{E}-11$ & 6233 & A & 0.8 & 133208 & 1.263E-09 & 4997 & A & 0.7 & 158485 & $6.857 \mathrm{E}-11$ & 8318 & A & 0.9 \\
\hline 107213 & $7.582 \mathrm{E}-11$ & 6317 & A & 0.6 & 134083 & $2.698 \mathrm{E}-10$ & 6567 & A & 0.6 & 159222 & $6.553 \mathrm{E}-11$ & 5708 & A & 0.8 \\
\hline 107259 & $8.167 \mathrm{E}-10$ & 8950 & A & 0.9 & 134169 & $2.329 \mathrm{E}-11$ & 5817 & A & 0.7 & 159561 & 3.665E-09 & 7883 & A & 0.8 \\
\hline 107582 & $1.427 \mathrm{E}-11$ & 5616 & A & 0.9 & 134439 & $7.962 \mathrm{E}-12$ & 5007 & A & 0.8 & 160693 & $1.235 \mathrm{E}-11$ & 5752 & A & 0.8 \\
\hline 108110 & $1.435 \mathrm{E}-10$ & 4580 & A & 0.9 & 134505 & $1.384 \mathrm{E}-09$ & 5058 & B & 0.9 & 161096 & $3.010 \mathrm{E}-09$ & 4601 & A & 0.6 \\
\hline 108153 & $1.088 \mathrm{E}-11$ & 4814 & A & 0.9 & 134987 & 7.413E-11 & 5591 & A & 1.0 & 161198 & $3.106 \mathrm{E}-11$ & 5268 & A & 1.0 \\
\hline 108255 & $3.218 \mathrm{E}-10$ & 5011 & A & 1.0 & 135153 & $3.350 \mathrm{E}-10$ & 6705 & B & 0.9 & 161797 & $1.222 \mathrm{E}-09$ & 5496 & A & 0.6 \\
\hline 108847 & 8.023E-12 & 4796 & A & 0.9 & 135379 & $6.283 \mathrm{E}-10$ & 8565 & B & 1.0 & 161892 & 2.171E-09 & 4471 & B & 0.9 \\
\hline 108954 & $8.748 \mathrm{E}-11$ & 6068 & A & 1.0 & 135502 & $2.313 \mathrm{E}-10$ & 8926 & A & 1.2 & 163588 & $1.281 \mathrm{E}-09$ & 4495 & A & 0.9 \\
\hline 109787 & 7.695E-10 & 8673 & B & 0.9 & 135722 & 1.360E-09 & 4810 & A & 0.7 & 163772 & 8.089E-11 & 8284 & A & 0.9 \\
\hline 110458 & $4.997 \mathrm{E}-10$ & 4728 & B & 0.9 & 136274 & $1.965 \mathrm{E}-11$ & 5331 & A & 0.9 & 163810 & $4.112 \mathrm{E}-12$ & 5595 & A & 0.9 \\
\hline 111397 & $1.590 \mathrm{E}-10$ & 9216 & A & 0.9 & 136352 & $1.519 \mathrm{E}-10$ & 5614 & B & 0.7 & 163917 & $1.536 \mathrm{E}-09$ & 4849 & B & 0.9 \\
\hline 111721 & $2.570 \mathrm{E}-11$ & 5165 & A & 0.5 & 138290 & $6.044 \mathrm{E}-11$ & 6858 & A & 0.6 & 163993 & $1.032 \mathrm{E}-09$ & 5011 & A & 0.7 \\
\hline 111812 & $3.098 \mathrm{E}-10$ & 5747 & A & 0.9 & 138716 & $4.989 \mathrm{E}-10$ & 4801 & B & 0.8 & 164507 & $9.068 \mathrm{E}-11$ & 5560 & A & 0.9 \\
\hline 111980 & $1.304 \mathrm{E}-11$ & 5667 & A & 0.8 & 139590 & $2.712 \mathrm{E}-11$ & 6116 & A & 0.9 & 165135 & $2.291 \mathrm{E}-09$ & 4773 & B & 1.2 \\
\hline 111996 & $6.453 \mathrm{E}-12$ & 4571 & A & 0.9 & 139663 & $4.555 \mathrm{E}-10$ & 4332 & B & 0.9 & 165341 & 7.992E-10 & 4983 & A & 0.7 \\
\hline 112164 & $1.213 \mathrm{E}-10$ & 5916 & B & 0.7 & 139798 & $1.270 \mathrm{E}-10$ & 6748 & A & 0.5 & 166620 & $9.115 \mathrm{E}-11$ & 4995 & A & 0.8 \\
\hline 113101 & $7.572 \mathrm{E}-12$ & 5481 & A & 0.9 & 140301 & $1.169 \mathrm{E}-10$ & 4661 & A & 0.9 & 166640 & $1.889 \mathrm{E}-10$ & 5079 & A & 1.2 \\
\hline 113314 & $3.426 \mathrm{E}-10$ & 9220 & B & 0.9 & 140573 & 3.353E-09 & 4558 & A & 0.6 & 167858 & $5.881 \mathrm{E}-11$ & 7050 & A & 0.6 \\
\hline 114606 & $9.197 \mathrm{E}-12$ & 5623 & A & 0.9 & 141003 & $9.186 \mathrm{E}-10$ & 8542 & A & 0.9 & 168009 & $8.352 \mathrm{E}-11$ & 5833 & A & 0.9 \\
\hline 114710 & $5.263 \mathrm{E}-10$ & 5959 & A & 0.5 & 141352 & $2.788 \mathrm{E}-11$ & 6390 & A & 1.2 & 169414 & $1.206 \mathrm{E}-09$ & 4460 & A & 0.7 \\
\hline 114762 & $3.269 \mathrm{E}-11$ & 5904 & A & 0.9 & 141653 & $2.457 \mathrm{E}-10$ & 8952 & A & 0.9 & 170845 & $4.809 \mathrm{E}-10$ & 4907 & B & 1.2 \\
\hline 114837 & $2.852 \mathrm{E}-10$ & 6143 & B & 0.9 & 141714 & $4.152 \mathrm{E}-10$ & 5247 & A & 0.9 & 171391 & $2.879 \mathrm{E}-10$ & 5116 & B & 1.2 \\
\hline 115383 & $2.201 \mathrm{E}-10$ & 5989 & B & 0.7 & 141891 & $1.914 \mathrm{E}-09$ & 7112 & B & 0.9 & 172310 & $1.236 \mathrm{E}-11$ & 5441 & A & 0.9 \\
\hline 115617 & $3.592 \mathrm{E}-10$ & 5430 & B & 0.7 & 142091 & $4.225 \mathrm{E}-10$ & 4731 & A & 0.9 & 172323 & $1.605 \mathrm{E}-11$ & 5814 & A & 0.9 \\
\hline 115892 & 2.247E-09 & 9102 & B & 0.9 & 142373 & $3.864 \mathrm{E}-10$ & 5745 & A & 0.6 & 173667 & $5.324 \mathrm{E}-10$ & 6329 & A & 0.7 \\
\hline 116842 & $6.443 \mathrm{E}-10$ & 8008 & A & 0.9 & 142575 & $1.019 \mathrm{E}-11$ & 6623 & A & 0.9 & 175190 & $4.602 \mathrm{E}-10$ & 4193 & B & 0.9 \\
\hline 117176 & $2.930 \mathrm{E}-10$ & 5482 & A & 0.6 & 143546 & $4.580 \mathrm{E}-10$ & 5079 & B & 0.9 & 175541 & $2.172 \mathrm{E}-11$ & 4979 & A & 0.9 \\
\hline 118098 & $1.166 \mathrm{E}-09$ & 8296 & B & 0.9 & 143761 & $1.875 \mathrm{E}-10$ & 5726 & A & 0.5 & 176411 & $8.600 \mathrm{E}-10$ & 4722 & A & 0.9 \\
\hline
\end{tabular}


Table 1. concluded

\begin{tabular}{|c|c|c|c|c|c|c|c|c|c|c|c|c|c|c|}
\hline $\mathrm{HD}$ & $\overline{\text { FLUX }}$ & TEMP & $\mathrm{S}$ & $\overline{\mathrm{PE}}$ & $\mathrm{HD}$ & $\overline{\text { FLUX }}$ & TEMP & $\bar{S}$ & $\mathrm{PE}$ & $\mathrm{HD}$ & $\overline{\text { FLUX }}$ & TEMP & $\mathrm{S}$ & $\overline{\mathrm{PE}}$ \\
\hline$\overline{176524}$ & $4.588 \mathrm{E}-10$ & 4555 & $\mathrm{~A}$ & 0.9 & 194598 & $1.242 \mathrm{E}-11$ & 5926 & $\mathrm{~A}$ & 0.6 & 211388 & $1.124 \mathrm{E}-09$ & 4140 & $\mathrm{~A}$ & 1.2 \\
\hline 176638 & $3.922 \mathrm{E}-10$ & 9710 & B & 0.9 & 195987 & $4.836 \mathrm{E}-11$ & 5059 & A & 0.9 & 211391 & $6.940 \mathrm{E}-10$ & 4985 & B & 0.7 \\
\hline 178253 & $6.352 \mathrm{E}-10$ & 8869 & B & 0.9 & 196171 & $1.959 \mathrm{E}-09$ & 4855 & B & 0.9 & 211476 & $4.168 \mathrm{E}-11$ & 5840 & A & 0.5 \\
\hline 178345 & $9.750 \mathrm{E}-10$ & 4575 & B & 1.2 & 197076 & $7.196 \mathrm{E}-11$ & 5774 & A & 0.6 & 212330 & $2.079 \mathrm{E}-10$ & 5613 & B & 0.9 \\
\hline 178428 & $1.035 \mathrm{E}-10$ & 5608 & A & 0.5 & 197692 & $5.637 \mathrm{E}-10$ & 6517 & B & 0.6 & 212943 & $4.531 \mathrm{E}-10$ & 4656 & A & 0.6 \\
\hline 179626 & $6.201 \mathrm{E}-12$ & 5761 & A & 0.9 & 197989 & $3.600 \mathrm{E}-09$ & 4732 & A & 0.6 & 213009 & $8.858 \mathrm{E}-10$ & 4884 & B & 0.9 \\
\hline 180711 & 2.007E-09 & 4793 & A & 1.2 & 198001 & $9.357 \mathrm{E}-10$ & 9227 & B & 0.9 & 214059 & $1.474 \mathrm{E}-11$ & 5553 & A & 0.9 \\
\hline 181276 & $9.826 \mathrm{E}-10$ & 4997 & A & 0.9 & 201381 & $5.118 \mathrm{E}-10$ & 5093 & B & 0.9 & 214868 & $7.600 \mathrm{E}-10$ & 4239 & A & 1.2 \\
\hline 181321 & $7.007 \mathrm{E}-11$ & 5710 & B & 0.9 & 201889 & $4.414 \mathrm{E}-11$ & 5621 & A & 0.6 & 214994 & $3.720 \mathrm{E}-10$ & 9443 & A & 0.9 \\
\hline 181597 & $1.210 \mathrm{E}-10$ & 4663 & A & 1.2 & 201891 & $1.709 \mathrm{E}-11$ & 5918 & A & 0.7 & 215182 & $2.092 \mathrm{E}-09$ & 5104 & A & 0.9 \\
\hline 181623 & $4.950 \mathrm{E}-10$ & 6778 & B & 0.9 & 203387 & $6.103 \mathrm{E}-10$ & 5105 & B & 0.9 & 215648 & $5.571 \mathrm{E}-10$ & 6222 & B & 0.9 \\
\hline 181655 & $8.369 \mathrm{E}-11$ & 5676 & A & 0.9 & 203504 & $8.644 \mathrm{E}-10$ & 4687 & A & 0.9 & 215721 & $2.663 \mathrm{E}-10$ & 4942 & B & 0.9 \\
\hline 182572 & $2.447 \mathrm{E}-10$ & 5528 & A & 0.5 & 203608 & $5.395 \mathrm{E}-10$ & 6073 & B & 0.7 & 216131 & $1.269 \mathrm{E}-09$ & 4981 & A & 0.5 \\
\hline 182807 & $8.929 \mathrm{E}-11$ & 6103 & A & 0.6 & 204121 & $9.207 \mathrm{E}-11$ & 6411 & B & 0.5 & 216627 & $1.322 \mathrm{E}-09$ & 8571 & B & 0.9 \\
\hline 184492 & $3.580 \mathrm{E}-10$ & 4694 & B & 0.9 & 203850 & $1.198 \mathrm{E}-11$ & 4780 & B & 0.9 & 216637 & $1.439 \mathrm{E}-10$ & 4446 & A & 0.9 \\
\hline 184499 & $6.227 \mathrm{E}-11$ & 5773 & A & 0.6 & 204577 & $6.532 \mathrm{E}-11$ & 6726 & B & 0.9 & 216735 & $3.393 \mathrm{E}-10$ & 9528 & A & 0.7 \\
\hline 184960 & $1.217 \mathrm{E}-10$ & 6220 & A & 0.7 & 204814 & $2.030 \mathrm{E}-11$ & 5374 & A & 0.9 & 216777 & $1.752 \mathrm{E}-11$ & 5634 & A & 0.9 \\
\hline 185395 & $4.008 \mathrm{E}-10$ & 6672 & A & 0.7 & 204867 & 2.040E-09 & 5474 & B & 0.5 & 216956 & 9.033E-09 & 8622 & B & 1.0 \\
\hline 185758 & $4.986 \mathrm{E}-10$ & 5415 & A & 0.7 & 205512 & $4.091 \mathrm{E}-10$ & 4696 & A & 0.6 & 217877 & $5.731 \mathrm{E}-11$ & 6011 & B & 1.0 \\
\hline 188114 & $8.675 \mathrm{E}-10$ & 4683 & B & 0.9 & 206453 & $4.104 \mathrm{E}-10$ & 5093 & B & 0.9 & 219080 & $3.847 \mathrm{E}-10$ & 7096 & A & 0.9 \\
\hline 188510 & $8.375 \mathrm{E}-12$ & 5636 & A & 0.9 & 209100 & $4.998 \mathrm{E}-10$ & 4577 & B & 0.8 & 219891 & $6.349 \mathrm{E}-11$ & 7856 & A & 1.2 \\
\hline 188512 & $1.024 \mathrm{E}-09$ & 4990 & A & 0.6 & 209750 & $2.085 \mathrm{E}-09$ & 5206 & B & 0.6 & 219953 & $9.256 \mathrm{E}-12$ & 5046 & A & 0.9 \\
\hline 189558 & $2.309 \mathrm{E}-11$ & 5666 & A & 0.7 & 209993 & $9.601 \mathrm{E}-11$ & 8617 & A & 0.7 & 220321 & $1.022 \mathrm{E}-09$ & 4602 & B & 0.9 \\
\hline 190299 & $2.757 \mathrm{E}-10$ & 4163 & A & 1.2 & 210027 & $8.020 \mathrm{E}-10$ & 6288 & A & 1.0 & 220594 & $6.996 \mathrm{E}-10$ & 4733 & B & 0.9 \\
\hline 190360 & $1.427 \mathrm{E}-10$ & 5580 & A & 0.5 & 210049 & $4.423 \mathrm{E}-10$ & 8847 & B & 0.9 & 220657 & $4.671 \mathrm{E}-10$ & 5942 & A & 0.7 \\
\hline 190404 & $3.975 \mathrm{E}-11$ & 5015 & A & 0.5 & 210144 & $2.285 \mathrm{E}-11$ & 5403 & A & 0.9 & 220957 & $9.539 \mathrm{E}-11$ & 4904 & A & 0.9 \\
\hline 190571 & $3.530 \mathrm{E}-11$ & 4987 & A & 0.9 & 210459 & $4.825 \mathrm{E}-10$ & 6249 & A & 0.9 & 221115 & $4.930 \mathrm{E}-10$ & 4985 & A & 0.9 \\
\hline 191277 & $2.912 \mathrm{E}-10$ & 4523 & A & 0.9 & 210460 & $9.820 \mathrm{E}-10$ & 5399 & A & 0.5 & 221914 & $2.537 \mathrm{E}-11$ & 5574 & A & 0.9 \\
\hline 192947 & $1.179 \mathrm{E}-09$ & 4933 & B & 0.5 & 210918 & $8.884 \mathrm{E}-11$ & 5658 & B & 0.9 & 222603 & $3.992 \mathrm{E}-10$ & 7766 & A & 0.9 \\
\hline 194013 & $2.680 \mathrm{E}-10$ & 4895 & B & 0.9 & 211006 & $1.828 \mathrm{E}-10$ & 4525 & A & 0.9 & 222935 & $1.375 \mathrm{E}-11$ & 5015 & A & 0.9 \\
\hline
\end{tabular}

\subsection{Determination of integrated flux}

To determine integrated stellar fluxes for the ISO calibration stars, designated $F L U X$, the techniques described by Blackwell et al. $(1990,1991)$ have been followed. An interstellar extinction correction was applied as described above. The final results, expressed in units of $10^{-9} \mathrm{Wm}^{-2}$, and corrected for interstellar extinction, are included in Table 1.

We relate the measured integrated fluxes, to values of $(B-V)_{0},(B 2-V 1)_{0},(b-y)_{0}$ and $(V-K)_{0}$, both as a check and as a tool for deriving fluxes in other circumstances where only such photometric quantities are available. With this in mind, Blackwell \& Petford (1991) have shown that a reasonably smooth relation exists between the quantity $F L U X 10^{0.4 V}$, denoted the reduced flux $R F L U X$, and polynomials in $(B-V)$ and $(V-K)$, with all quantities corrected for interstellar extinction. In deriving such polynomial relations in the present work we have discarded the apparently anomalous results for five stars, HD 10700, HD 20794, HD 130163, HD 209100 and HD 216956.
Table 2 summarises polynomial relations of the form $R F L U X=A+B x+C x^{2}+D x^{3}$ where $x$ is $(B-V)_{0}$, $(B 2-V 1)_{0},(V-K)_{0}$ or $(b-y)_{0}$ derived for all stars in Table 1 (excepting those with a small value of $[\mathrm{Fe} / \mathrm{H}]$ ). In the case of $(V-K)_{0}$, only stars observed by Johnson et al. (1966) have been used. The table includes standard deviations for the differences between observed and calculated values of $R F L U X$. These are reasonably small, but as mentioned above, a closer relationship cannot be expected because of the probable presence of unresolved binaries and metallicity effects as yet unaccounted for. Separate polynomials are given for Classes V, IV and III. Use of the extinction model of Arenou et al. (1992) yields a small increase in dispersion over that found using $A_{V}=0.8 \mathrm{mag} / \mathrm{kpc}$. The effect of metallicity may be included in the calculation of RFLUX by multiplying the appropriate polynomial given in Table 2 by the factor $(1-(0.16-1.9[\mathrm{Fe} / \mathrm{H}]) / 100)$. The importance of metallicity has been stressed by Alonso et al. (1996a). However, as the derived temperatures depend on the fourth root of the integrated flux, the effect is relatively small. Figure 1 shows the relation between $R F L U X$ and $(B-V)_{0}$ for 
Table 2. Coefficients in polynomial representations of $R F L U X$ in terms of photometric variables $(B-V),(B 2-V 1)$, $(V-K)$ and $(b-y)$, all corrected for interstellar extinction; $\sigma$ is the percentage standard deviation about the regression line. See text for correction for metallicity $[\mathrm{Fe} / \mathrm{H}]$

\begin{tabular}{lrrrrr}
\hline \multicolumn{1}{c}{$A$} & \multicolumn{1}{c}{$B$} & \multicolumn{1}{c}{$C$} & \multicolumn{1}{c}{$D$} \\
\hline Class V & & & & & \\
$(B-V)$ & 296.66 & -254.06 & 377.61 & -54.72 & 5.5 \\
$(B 2-V 1)$ & 263.27 & -130.17 & 411.37 & -1.10 & 7.0 \\
$(V-K)$ & 257.31 & -3.95 & -24.26 & 16.81 & 4.5 \\
$(b-y)$ & 290.60 & -397.69 & 905.00 & -71.96 & 5.6 \\
\hline Class IV & & & & \\
$(B-V)$ & 315.90 & -180.62 & 112.14 & 90.01 & 4.4 \\
$(B 2-V 1)$ & 280.22 & -177.64 & 409.75 & -0.82 & 3.0 \\
$(b-y)$ & 312.74 & -327.63 & 557.04 & & 4.8 \\
\hline Class III & & & & \\
$(B-V)$ & 260.40 & -72.82 & 90.77 & 63.56 & 4.3 \\
$(B 2-V 1)$ & 250.49 & 5.84 & 54.86 & 210.10 & 4.9 \\
$(V-K)$ & 248.56 & -2.57 & -4.88 & 10.23 & 2.9 \\
$(b-y)$ & 317.38 & -450.61 & 909.93 & -179.79 & 8.8 \\
\hline
\end{tabular}

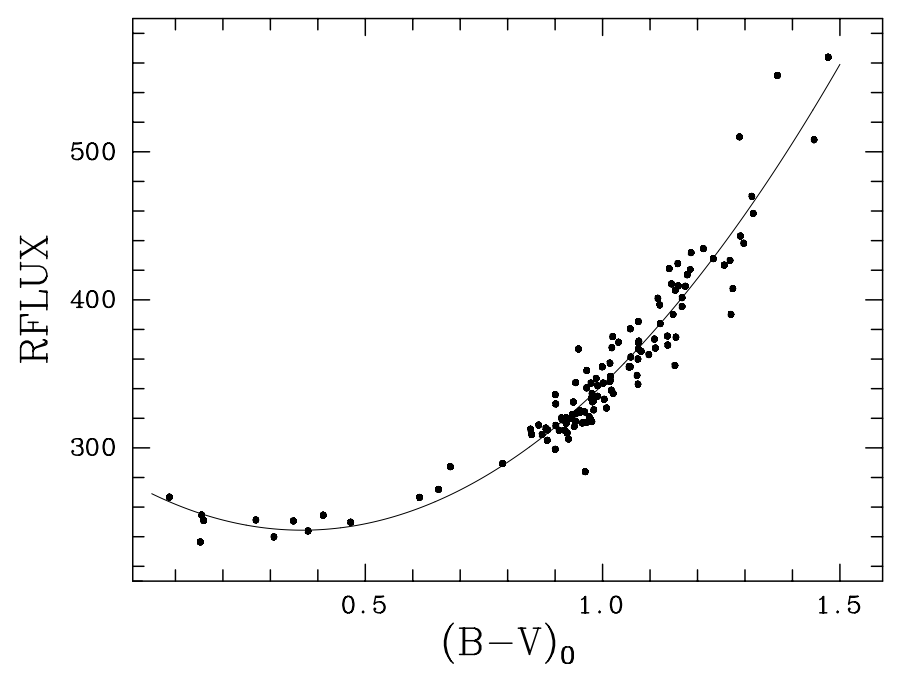

Fig. 1. Plot of reduced integrated flux, $R F L U X$, for Class III stars of known small metallicity against $(B-V)_{0} . R F L U X$ has been has been corrected for interstellar extinction; the standard deviation about the regression line is 4.3 per cent

Class III stars of small metallicity; the standard deviation about the regression line is 4.3 per cent.

\section{The determined temperatures}

Table 1 lists the final temperatures (denoted TEMP) derived for the chosen stars. The column $S$ shows the source of infrared data as described in the Table 1 caption. Where data have been obtained at one observatory only, these final temperatures are the means of several determinations based on either $J, K, L$ filters or $J, H, K, L$ filters. Table 3 shows the dependence of determined temperatures on the three infrared filters used at the two observatories. In all cases, the agreeement between the stellar temperatures determined using the three filters is close to one per

Table 3. Dependence of derived mean temperatures on adopted filters, with standard deviations of temperature. NB refers to a combination of published narrow band data (see text) with TCS narrow band data

\begin{tabular}{cccc}
\hline Filter ratios & \multirow{3}{*}{ ESO } & Temperature ratios & NB \\
& & & TCS \\
\hline$J / K$ & $1.001 \pm 0.011$ & $1.003 \pm 0.002$ & $1.011 \pm 0.016$ \\
$H / K$ & $0.993 \pm 0.011$ & & $1.008 \pm 0.075$ \\
$L / K$ & $0.999 \pm 0.009$ & $0.992 \pm 0.009$ & $0.997 \pm 0.007$ \\
\hline
\end{tabular}

cent. We show later that temperatures derived from TCS narrow band measures agree well with published temperatures of Blackwell \& Lynas-Gray (1994), obtained using very similar filters. Because the TCS narrow band temperatures and published narrow band temperatures have equal weight, their averages are presented in Table 1 as final temperatures. Estimates of the percentage error of all temperature determinations are tabulated in the column $\mathrm{PE}$ of Table 1. These estimates are based on considerations concerning the accuracy of absolute fluxes and the agreement between temperatures derived using the various filters, and on the relations between temperatures and various photometric indices discussed below. No account has been taken of uncertainties due to difficulties in the theoretical modelling of stellar atmospheres discussed for example by Mégessier (1994). In addition, important stellar data such as metallicity may either be unavailable or inaccurate. Because of these considerations, the reliability of such estimates of absolute accuracy may be regarded as questionable, but we suggest that they give at least a useful indication of the relative accuracies of the determined stellar temperatures presented in Table 1. Unfortunately, it is not possible to make a definitive comparison between temperatures determined using interferometrically measured angular diameters and integrated fluxes because such determinations are most often made using low temperature giant stars, and the IRFM technique (as presented in this paper) is not suited to such low temperature stars because infrared fluxes at longer wavelengths would be needed.

\section{Tests for reliability of determined temperatures}

It is important to attempt an assessment of the reliability of the determined temperatures and, in an initial test, we relate them to several photometric parameters that are sensitive to temperature. An advantage of this method is that it subsequently also makes available a large body of new stellar temperatures, of good accuracy, through 
the use of a database of the appropriate stellar parameters. The parameters used are $(B 2-V 1)_{0},(b-y)_{0}$ and $(B-V)_{0}$, taken from the literature and corrected for interstellar extinction as already described. A further potentially important quantity is $(V-K)_{0}$, especially because, as pointed out by Carney et al. (1994), this parameter is not greatly sensitive to metallicity. However, it is less useful because the present infrared data are based partly on three distinct systems for $K$, which can only be interchanged with difficulty, so tests must be confined to each of the three systems separately. Because it is likely that such temperature/colour relations depend slightly on stellar luminosity, we discuss in Sect. 5 the results for the two broad luminosity classes III and V separately, beginning with the published narrow band measures and the narrow band measures contained in the present new data base of infrared measures.

\section{Use of narrow band data (NB)}

\subsection{Temperatures for luminosity class III stars}

To assess the reliability of the ISO calibration temperatures, we compare the published temperatures (Blackwell et al. 1990; Blackwell \& Lynas-Gray 1994) using narrow band data, with the temperatures found using TCS narrow band data, discussed in the present paper. The mean difference between the published and the completely independent temperatures presented here is 0.05 per cent with a standard deviation of 0.75 per cent. This is reasonably satisfactory, and we therefore consider it justifiable to take the average of the three (or occasionally two) temperature determinations for Class III stars, i.e. those published and the new NB temperatures, and relate them to photometric colours. The results are presented in Table 4, which shows polynomial coefficients together with respective percentage standard deviations from the regression curve for $(B 2-V 1),(b-y),(V-K)$ and $(B-V)$, all corrected for interstellar extinction. The table suggests that $(B 2-V 1)$ may be considered a good indicator of temperature, as Hauck (1994) has emphasised, and that it is clearly useful for obtaining reliable photometric estimates of temperature. Figure 2 shows the close relation between temperature and $(V-K)_{0}$.

\subsection{Temperatures for luminosity class $V$ stars using narrow band data}

A comparison between the average of published temperatures (Blackwell et al. 1990; Blackwell \& Lynas-Gray 1994) with the new ISO calibration star temperatures shows a mean difference of 0.43 per cent with a standard deviation of 1.06 per cent. This suggests that the two batches of data may be combined. The regression constants for the combined data are shown in Table 4 together with standard deviation values. The standard deviations are comparable
Table 4. Regression Coefficients $A, B, C, D$ for relations between stellar temperature $T$ and values of designated photometric parameters $p: T=A+B p+C p^{2}+D p^{3} ; \sigma$ is the corresponding percentage standard deviation in temperature and $\mathrm{M}$ the measurement code noted below

\begin{tabular}{|c|c|c|c|c|c|c|c|}
\hline Class & $\mathrm{M}$ & $p$ & $A$ & $B$ & $C$ & $D$ & $\sigma$ \\
\hline III & 1 & $\begin{array}{c}(B 2-V 1) \\
(b-y) \\
(V-K) \\
(B-V)\end{array}$ & $\begin{array}{l}7626 \\
8757 \\
8970 \\
8925\end{array}$ & $\begin{array}{l}-6841 \\
-11242 \\
-2970 \\
-7664\end{array}$ & $\begin{array}{l}5514 \\
10676 \\
626 \\
4867\end{array}$ & $\begin{array}{l}-2293 \\
-4616 \\
-53 \\
-1339\end{array}$ & $\begin{array}{l}0.82 \\
1.04 \\
0.73 \\
1.55\end{array}$ \\
\hline $\mathrm{V}$ & 1 & $\begin{array}{c}(B 2-V 1) \\
(b-y) \\
(V-K) \\
(B-V)\end{array}$ & $\begin{array}{l}7675 \\
8870 \\
9137 \\
8973\end{array}$ & $\begin{array}{l}-6847 \\
12789 \\
-3496 \\
-7908\end{array}$ & $\begin{array}{l}7819 \\
20418 \\
1063 \\
6087\end{array}$ & $\begin{array}{l}-7967 \\
-19250 \\
-158 \\
-2575\end{array}$ & $\begin{array}{l}0.67 \\
0.67 \\
0.82 \\
1.04\end{array}$ \\
\hline III & 2 & $\begin{array}{c}(B 2-V 1) \\
(b-y) \\
(V-K) \\
(B-V)\end{array}$ & $\begin{array}{l}7617 \\
8512 \\
9052 \\
8546\end{array}$ & $\begin{array}{l}-5847 \\
-8871 \\
-3032 \\
-5616\end{array}$ & $\begin{array}{l}2881 \\
4743 \\
672 \\
2139\end{array}$ & $\begin{array}{l}-430 \\
-343 \\
-66 \\
-234\end{array}$ & $\begin{array}{l}1.51 \\
1.44 \\
0.58 \\
1.15\end{array}$ \\
\hline $\mathrm{V}$ & 3 & $\begin{array}{c}(B 2-V 1) \\
(b-y) \\
(V-K) \\
(B-V)\end{array}$ & $\begin{array}{l}7738 \\
9017 \\
9352 \\
9139\end{array}$ & $\begin{array}{l}-7832 \\
-12771 \\
-3583 \\
-8853\end{array}$ & $\begin{array}{l}7594 \\
14831 \\
974 \\
6558\end{array}$ & $\begin{array}{l}-4414 \\
-9791 \\
-117 \\
2163\end{array}$ & $\begin{array}{l}1.04 \\
1.27 \\
0.57 \\
1.54\end{array}$ \\
\hline $\mathrm{V}$ & 4 & $\begin{array}{c}(B 2-V 1) \\
(b-y) \\
(V-K) \\
(B-V)\end{array}$ & $\begin{array}{l}7131 \\
8151 \\
8831 \\
7404\end{array}$ & $\begin{array}{l}-4273 \\
-7025 \\
-2724 \\
-3029\end{array}$ & $\begin{array}{l}675 \\
1815 \\
501 \\
286\end{array}$ & -33 & $\begin{array}{l}2.30 \\
2.31 \\
0.73 \\
2.74\end{array}$ \\
\hline III & 5 & $\begin{array}{c}(B 2-V 1) \\
(b-y) \\
(V-K) \\
(B-V)\end{array}$ & $\begin{array}{l}7627 \\
8579 \\
8723 \\
8826\end{array}$ & $\begin{array}{l}-6369 \\
-9288 \\
-2516 \\
-7197\end{array}$ & $\begin{array}{l}4229 \\
5005 \\
352 \\
4400\end{array}$ & -1201 & $\begin{array}{l}1.14 \\
0.96 \\
1.30 \\
0.70\end{array}$ \\
\hline $\mathrm{V}$ & 6 & $\begin{array}{c}(B 2-V 1) \\
(b-y) \\
(V-K) \\
(B-V)\end{array}$ & $\begin{array}{l}7876 \\
9054 \\
8326 \\
9133\end{array}$ & $\begin{array}{l}-7806 \\
-11539 \\
-2153 \\
-7753\end{array}$ & $\begin{array}{l}4886 \\
7535 \\
260 \\
3468\end{array}$ & & $\begin{array}{l}2.23 \\
2.59 \\
0.78 \\
1.54\end{array}$ \\
\hline \multicolumn{8}{|c|}{$\begin{array}{l}\text { Notes: } \\
1 \text { - Narrow Band Average } \\
2 \text { - Average of TCS and Narrow Band } \\
3 \text { - TCS only }(6000 \mathrm{~K} \leq T \leq 9000 \mathrm{~K}) \\
4 \text { - TCS only }(4200 \mathrm{~K} \leq T \leq 6000 \mathrm{~K}) \\
5 \text { - ESO only }(4500 \mathrm{~K} \leq T \leq 7000 \mathrm{~K}) \\
6 \text { - ESO only }(4600 \mathrm{~K} \leq T \leq 6000 \mathrm{~K}) \text {. }\end{array}$} \\
\hline
\end{tabular}

with those for Class III stars presented in the same table. Figure 3 shows the good relation that exists between temperature and $(V-K)_{0}$. There is no significant improvement in standard deviation for either class when the chosen temperature range is smaller.

A comparison of polynomials for Class III and V star temperatures obtained using narrow band data shows a real difference between the derived temperatures for a given colour, of order 5 per cent.

\section{Combination of temperature determinations}

There are 8 stars with temperatures determined using both TCS data and published narrow band data. For these 


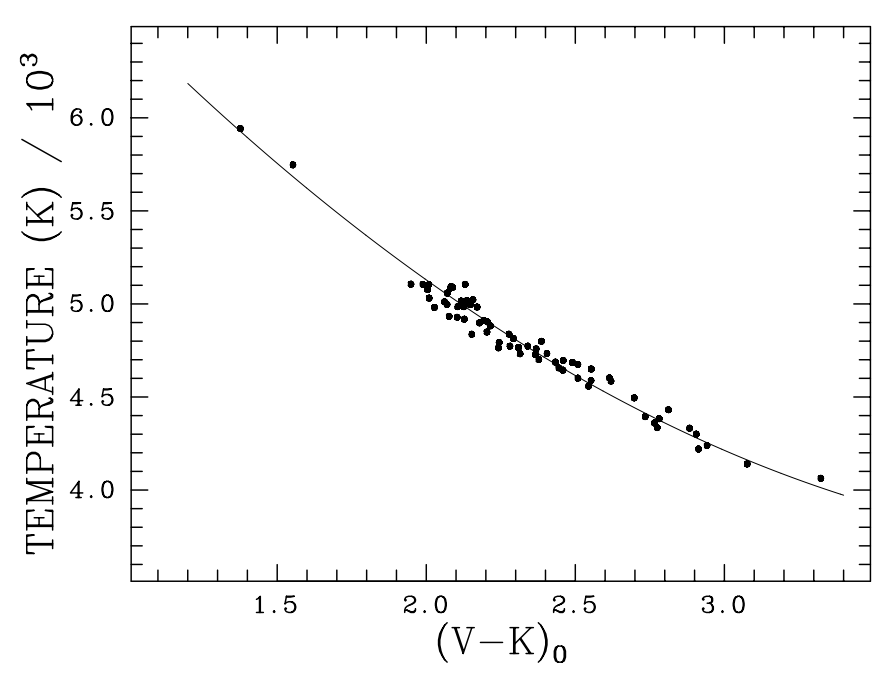

Fig. 2. Plot of temperature of Class III stars against $(V-K)_{0}$ where $K$ is on the Johnson scale

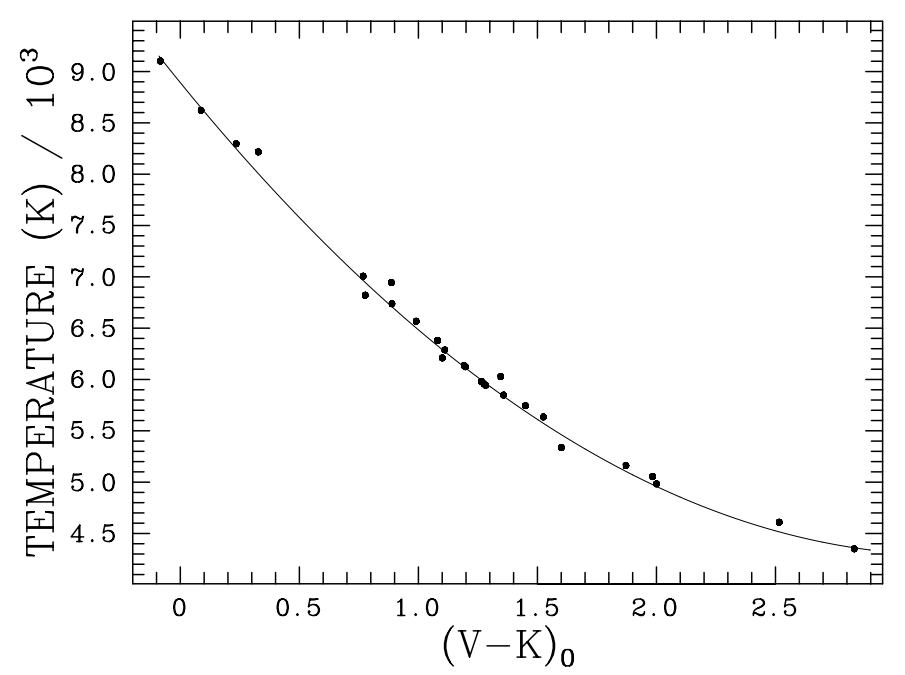

Fig. 3. Plot of temperature of Class $\mathrm{V}$ stars against $(V-K)_{0}$ where $K$ is on the Johnson scale

stars, the mean difference in determined temperatures is 0.33 per cent, with a standard deviation of 1.43 per cent. These differences may be regarded as satisfactory, and we have taken an average of the two sets of temperatures for these overlapping 8 stars, to be included in the total of Class III stars using TCS data. The regression relations between temperature and photometric parameters for all these stars, together with the standard deviations, are included in Table 4.

Unlike the situation for Class III stars, there are few overlapping published data for Class V stars, so we use the TCS data alone. The regression relations between temperatures in the two ranges $6000 \mathrm{~K}$ to $9500 \mathrm{~K}$, and $4200 \mathrm{~K}$ to $6000 \mathrm{~K}$, and photometric parameters are included in Table 4. Discounting the relation with $(V-K)_{0}$, the most useful parameter for representation of temperature is $(B 2-V 1)_{0}$, with a standard deviation of 1.04 per cent.

Relations between photometric parameters and temperatures for all ESO stars of Class III and Class V are included in Table 4.

\section{Assessment of reliability of final results}

\subsection{Comparison of direct measures of temperature}

Some assessment of reliability can be made by a direct comparison of temperatures determined independently using the three main sources of data; ESO, TCS and narrow band, to which may be added temperatures published using narrow band data. The relevant temperature ratios, ESO/TCS, TCS/Published NB are given in Table 5, together with standard deviations and the number of stars in common. The inference from these comparisons is that the temperature scales given by the three independent sources of data (narrow band, TCS and ESO) for overlapping stars are not significantly different.

Table 5. Ratios of temperatures found for ESO and TCS stars, and TCS and Published NB stars, with standard deviations

\begin{tabular}{|cccc|}
\hline Class & No. of stars & $T(\mathrm{ESO}) / T(\mathrm{TCS})$ & $\sigma$ \\
III & 11 & 1.005 & 0.013 \\
V & 6 & 1.004 & 0.003 \\
& & & \\
\hline Class & No. of stars & $T(\mathrm{TCS}) / T($ PublishedNB $)$ & $\sigma$ \\
III & 8 & 1.006 & 0.014 \\
V & 7 & 1.010 & 0.023 \\
& & & \\
Class & No. of stars & $T(\mathrm{NB}) / T($ PublishedNB $)$ & $\sigma$ \\
All & 65 & 0.999 & 0.010 \\
& & & \\
\hline
\end{tabular}

\subsection{Comparison of temperatures derived from photometric parameters}

A further test of reliability is a comparison of the several relations that have been derived for temperature versus the photometric parameters, $(B 2-V 1),(b-y),(B-V)$ and $(V-K)$, corrected for extinction. The result is that the average of the ESO, class $\mathrm{V}$, temperatures for these parameters differs from the average of the TCS, Class V, temperatures by 0.28 per cent $(\sigma=0.72$ per cent). For Class III stars, the averages differ by 1.31 per cent $(\sigma=$ 0.79 per cent). As these comparisons have been made using pairs of star lists that have so few stars in common, it is not possible to comment on the significance or otherwise of any difference between temperatures derived from ESO and TCS data. 


\section{Final determinations of temperature and their relation to photometric indices}

Now that it has been established that any differences between temperatures found using TCS, ESO, narrow band and narrow band published filters, are reasonably small, we combine together all of the various measured temperatures for each star to give the results listed in Table 1 . In this section we relate these temperatures to stellar photometry parameters. We have so far neglected the small additional effect of metallicity in these representations of temperatures and a small correction term for this will be discussed later in Sect. 9.

\subsection{Use of photometric parameters in the visible region}

For this purpose we divide the stars of Table 1 into the three luminosity classes V, IV and III, and consider them separately, beginning with class V. Further, because we wish to compute polynomial coefficients of good accuracy for use in deriving temperatures from a database of photometric parameters, we choose for this purpose only the measures from Table 1 that we judge on independent evidence to be the most reliable. This segregation is based on the extent of the agreement among measures using different filters, agreement with previous measures using the IRFM, and the existence of a complete MK spectral type. These stars have temperatures accurate to between 0.5 per cent and 0.8 per cent in Table 1 . Also, as our wish is to derive an accurate representation of stellar temperatures by a polynomial and a small correction factor for the effect of metallicity, we include at this stage only those stars with a measured metallicity that is less than 0.2 dex. In the following sections, we present separate regression analyses using data for the $(B 2-V 1),(B-V)$ and $(b-y)$ photometric parameters, all corrected for interstellar extinction, for the three luminosity classes V, IV and III separately, and for the two temperature regions, $T<6000 \mathrm{~K}$ and $T>6000 \mathrm{~K}$, where this is possible, and including the restrictions described above.

\subsection{Polynomial relations for stars of class $V, I V$ and $I I I$}

Table 6 presents the polynomial coefficients for relations between temperature and the photometric quantities $(B 2-V 1)_{0},(B-V)_{0}$ and $(b-y)_{0}$, derived in the way described above for these classes of stars that have very small metallicities. Standard deviations in the percentage differences between measured and calculated temperatures are also given. These standard deviations may be taken as a guide to the reliability of temperature determinations for the selected stars, but it is likely that the deviations also include a small spread in the determinations of each photometric parameter, together with a real spread in stellar temperatures. Figure 3 shows a typical plot of temperature versus $(V-K)_{0}$ for class $\mathrm{V}$ stars, but the best parameter for all class $\mathrm{V}$ stars is apparently $(b-y)_{0}$. For class
Table 6. Regression Coefficients $A, B, C$ for relations between stellar temperature $T$ and values of designated photometric parameters $p: T=A+B p+C p^{2}$ for final temperature determinations; $\sigma$ is the corresponding percentage standard deviation in temperature

\begin{tabular}{lcrrrr}
\hline & $p$ & $\mathrm{~A}$ & $\mathrm{~B}$ & $\mathrm{C}$ & $\sigma$ \\
\hline Class V & $(B 2-V 1)$ & 7995 & -7246 & 3284 & 1.52 \\
$T<6000 \mathrm{~K}$ & $(b-y)$ & 9865 & -13319 & 7483 & 1.30 \\
& $(B-V)$ & 8515 & -5270 & 1440 & 1.72 \\
Class V & $(B 2-V 1)$ & 7749 & -7911 & 7856 & 1.20 \\
$T>6000 \mathrm{~K}$ & $(b-y)$ & 9034 & -12562 & 11813 & 1.09 \\
& $(B-V)$ & 9134 & -8600 & 5398 & 1.32 \\
Class V & & & & & \\
$4000 \mathrm{~K}<T<9000 \mathrm{~K}$ & $(V-K)$ & 8921 & -2848 & 441 & 1.30 \\
& & & & & \\
Class IV & $(B 2-V 1)$ & 7949 & -6944 & 3407 & 0.91 \\
$T>5000 \mathrm{~K}$ & $(b-y)$ & 9030 & -11396 & 8313 & 1.10 \\
& $(B-V)$ & 9078 & -6545 & 2298 & 1.43 \\
Class III & & & & & \\
$T<6000 \mathrm{~K}$ & $(B 2-V 1)$ & 5504 & -190 & -1198 & 1.01 \\
& $(b-y)$ & 4507 & 3213 & -4454 & 1.00 \\
Class III & $(B-V)$ & 4842 & 1413 & -1426 & 0.91 \\
$T>6000 \mathrm{~K}$ & $(B 2-V 1)$ & 7581 & -5927 & 3898 & 1.20 \\
& $(b-y)$ & 8613 & -10332 & 8613 & 0.99 \\
Class III & $(B-V)$ & 8667 & -6383 & 3145 & 1.36 \\
$4000 \mathrm{~K}<T<9000 \mathrm{~K}$ & $(V-K)$ & 8305 & -2036 & 224 & 1.38 \\
\hline & & & & & \\
\hline & & & & &
\end{tabular}

IV stars, regression constants and standard deviations for class IV stars are given only for temperatures greater than $5000 \mathrm{~K}$. The restrictions described above have reduced the number of available stars so much that a division into two temperature ranges is not practical. However, a plot of temperature against $(B 2-V 1)_{0}$ shows that a discussion of the whole range of temperature for these stars is reasonable, and all available stars have been used for the calculation of regression coefficients presented in Table 6 . The standard deviation of temperatures, of 0.91 per cent, is satisfactory. Regression constants for class III stars, using the above restrictions, are given for temperatures in the two ranges $T<6000 \mathrm{~K}$ and $T>6000 \mathrm{~K}$. The quantity $(V-K)_{0}$, where $K$ is measured on the Johnson scale, is especially useful because it usually yields a standard deviation about the regression line that is smaller than those shown by other photometric parameters.

\section{The importance of metallicity for temperature determination}

We have taken account of the the effect of metallicity on temperature determination for all of the stars in the present study for which direct measurements of $[\mathrm{Fe} / \mathrm{H}] \mathrm{ex}-$ ist. Unfortunately, measures are available for only 35 per cent of the total number of stars in the programme, and 
this is reflected to some extent in the spread of the relations between temperature and photometric indices such as $(B 2-V 1)_{0}$. Figure 4 plots derived temperatures against

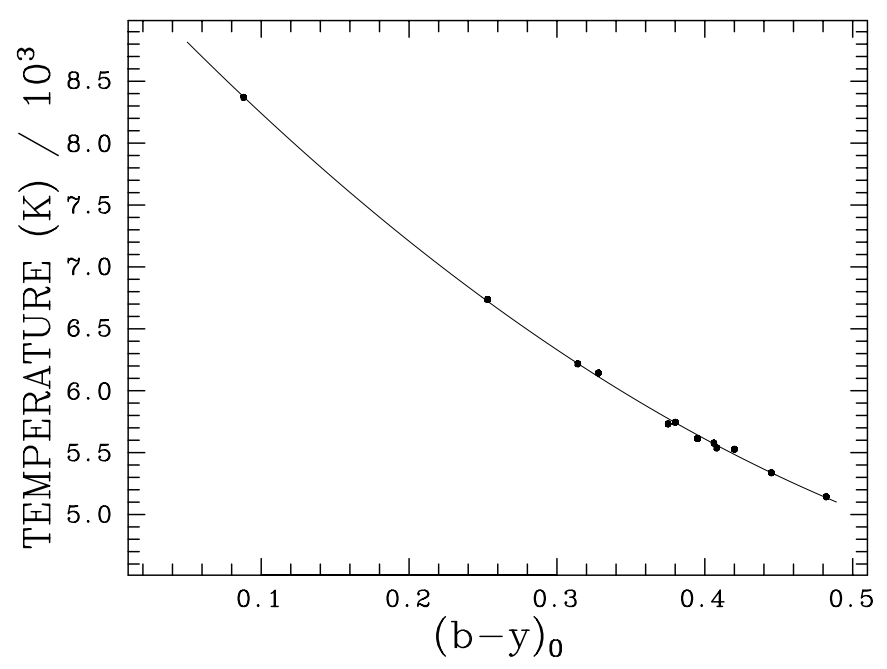

Fig. 4. Plot of temperatures of Class $\mathrm{V}$ stars against $(b-y)_{0}$. The stars have known metallicities in the range $-0.59<[\mathrm{Fe} / \mathrm{H}]<-0.34$

the arbitarily selected parameter $(b-y)_{0}$, using class $\mathrm{V}$ stars of the higher class of quality, with known values of $[\mathrm{Fe} / \mathrm{H}]$ in the narrow range $-0.59<[\mathrm{Fe} / \mathrm{H}]<-0.34$. In these circumstances, the spread about the regression line is very small, although it must be admitted that there are only a few points.

In order to derive the effect of metallicity on the representation of determined temperature by functions of photometric indices, we have evaluated the residuals in a regression as a function of measured metallicity $[\mathrm{Fe} / \mathrm{H}]$. For stars of luminosity classes $\mathrm{V}$ and IV, the residuals depend on metallicity according to the function $(0.40+1.30[\mathrm{Fe} / \mathrm{H}])$ per cent. Accordingly, where metallicity values exist, the polynomial relations derived in the previous sections should be increased by this function. The full expression for temperature determination using a photometric quantity $x$, where $x$ can be $(b-y)_{0},(B 2-V 1)_{0}$ or $(B-V)_{0}$, and metallicity $[\mathrm{Fe} / \mathrm{H}]$ is now:

$T=\left(A+B x+C x^{2}\right)(1+(0.40+1.30[\mathrm{Fe} / \mathrm{H}]) / 100)$.

\section{Comparisons with other temperature determinations}

Bell \& Gustafsson (1989) have determined temperatures for 95 stars using both synthetic infrared colours and the IRFM, using the Johnson $K$-band. Mean temperatures found using these two methods are listed by these authors as "adopted" temperatures, which we suggest is the more significant for a comparison because they partly depend on a different technique. Table 7 shows a comparison between these "adopted" temperatures and the present IRFM temperatures for 33 stars. The mean difference $T$ (Oxford-BG) is -0.41 per cent with a standard deviation of 1.95 per cent. The results of comparisons with the results obtained by other authors are summarised in Table 8 .

Table 7. Comparison of ISO temperatures ( $T$ (ISO)) with the adopted temperatures ( $T(\mathrm{BG}))$ determined by Bell \& Gustafsson (1989); $\Delta$ gives the percentage difference $T(\mathrm{ISO})-T(\mathrm{BG})$

\begin{tabular}{rrrrrrrr}
\hline HR & $T$ (ISO) & $T(\mathrm{BG})$ & $\Delta$ & HR & $T$ (ISO) & $T(\mathrm{BG})$ & $\Delta$ \\
\hline 434 & 4063 & 4046 & 0.42 & 5602 & 4997 & 4929 & 1.36 \\
464 & 4360 & 4425 & -1.49 & 5681 & 4810 & 4832 & -0.46 \\
753 & 4759 & 4775 & -0.34 & 5777 & 4801 & 4742 & 1.23 \\
996 & 5713 & 5650 & 1.10 & 5854 & 4558 & 4321 & 5.20 \\
1325 & 5161 & 5114 & 0.91 & 5889 & 5247 & 5608 & -6.88 \\
1409 & 4911 & 4911 & 0.00 & 5901 & 4731 & 4920 & -3.99 \\
1464 & 5015 & 4974 & 0.82 & 6220 & 4918 & 4913 & 0.10 \\
1907 & 4701 & 4719 & -0.38 & 6603 & 4601 & 4603 & -0.04 \\
2077 & 4764 & 4831 & -1.41 & 6623 & 5496 & 5527 & -0.56 \\
2990 & 4837 & 4896 & -1.22 & 7479 & 5415 & 5429 & -0.26 \\
3403 & 4524 & 4542 & -0.40 & 7602 & 4990 & 5098 & -2.16 \\
4335 & 4650 & 4542 & 2.32 & 8085 & 4350 & 4463 & -2.60 \\
4883 & 5747 & 5761 & -0.24 & 8255 & 4696 & 4715 & -0.06 \\
4983 & 5959 & 6024 & -1.09 & 8499 & 4985 & 4956 & 0.58 \\
5019 & 5430 & 5650 & -0.06 & 8684 & 4981 & 5044 & -1.22 \\
5429 & 4220 & 4303 & -1.97 & & & & \\
\hline
\end{tabular}

Table 8. Comparisons between ISO calibration star temperatures ( $T(\mathrm{ISO})$ - this work) and other determinations $(T(\mathrm{OTHER})) ; \quad \Delta$ gives the percentage difference $T$ (ISO) $-T$ (OTHER)

\begin{tabular}{lcr}
\hline Publication & No. of Stars & \multicolumn{1}{c}{$\Delta$} \\
\hline Alonso et al. (1996b) & 93 & $0.12 \pm 1.25$ \\
Bell \& Gustafsson (1989) & 33 & $-0.41 \pm 1.95$ \\
Fuhrmann et al. (1994) & 13 & $0.69 \pm 1.96$ \\
Saxner \& Hammarbäck (1985)* & 30 & $-0.46 \pm 0.89$ \\
Glushneva et al. (1993)** & 3 & $-0.33 \pm 0.62$ \\
Peterson \& Carney (1979) & 10 & $0.62 \pm 2.13$
\end{tabular}

* omitting discrepant temperature determination for HR 2085
** omitting discrepant temperature determination for HR 5986 .

\section{Conclusions}

We have been concerned here with the interpretation of data to give stellar temperatures that are as accurate as we can manage, and with an attempt at assessing their relative and absolute accuracies as reliably as possible. No account has been taken of inaccuracies in the original data, for we judge that the largest contributions to errors in the final temperatures originate in the theoretical interpretion 
of data. The derivation of monochromatic fluxes from observations made using wide wavelength bands is difficult and uncertain, and may introduce unacceptably large errors which depend on uncertain line strengths. Our judgement of the relative accuracies of the final temperatures is reasonably well based and may be regarded as reliable. However, the more difficult task is the assessment of final absolute probable errors. Some contributions to these errors are clear. For example, $[\mathrm{Fe} / \mathrm{H}]$ values are not available for many stars in the observed list, and even the solar iron abundance is now regarded as uncertain (Blackwell et al. $1995)$; where no $[\mathrm{Fe} / \mathrm{H}]$ value is available, we have given an accuracy of 0.9 per cent. A theoretically derived atmosphere is needed, and although these are now more reliable than formerly, some uncertainty about their use remains. An important and extensive investigation of the accuracy of model predictions has been made by van der Bliek et al. (1996), following on the earlier work by Mégessier (1994). In the present paper we suggest that the wholly empirical use of plots of temperature against intrinsic photometric indices, e.g. $(V-K)_{0}$ and $(b-y)_{0}$, provide useful evidence for relative accuracies, whilst comparisons with measures made by a variety of methods are useful for detecting gross errors.

Acknowledgements. We are indebted to the ISO Ground Based Preparatory Programme which has entrusted us with the work reported here. Rapid progrsss would not have been possible without the generosity of Drs. van der Bliek and P.L. Hammersley, who kindly made their observations available in advance of publication. Hipparcos parallaxes were provided by Dr. Perryman, also in advance of publication. Dr. Marie Jourdain de Muizon gave constant and patient help during the investigation and the authors are especially grateful to her. Obtaining infrared monochromatic flux densities would have been more difficult without Dr. Hammersley's advice. The authors have also benefitted from correspondence with Professor G.P. di Benedetto. Comments and suggestions by an anonymous referee have contributed to the present paper.

\section{References}

Allen C.W., 1955, Astrophysical Quantities. Athlone Press Alonso A., Arribas S., Martínez-Roger C., 1995, A\&A 297, 197 Alonso A., Arribas S., Martínez-Roger C., 1996a, A\&A 313, 873

Alonso A., Arribas S., Martínez-Roger C., 1996b, A\&AS 117, 227

Arenou F., Grenon M., Gomez A., 1992, A\&A 258, 104

Bell R.A., Gustafsson B., 1989, MNRAS 236, 653

Blackwell D.E., Lynas-Gray A.E., 1994, A\&A 282, 899

Blackwell D.E., Petford A.D., 1991, A\&A 250, 459

Blackwell D.E., Petford A.D., Shallis M.J., 1980, A\&A 82, 249

Blackwell D.E., Petford A.D., Arribas A., Haddock D.J., Selby M.J., 1990, A\&A 232, 396
Blackwell D.E., Lynas-Gray A.E., Petford A.D., 1991, A\&A 245,567

Blackwell D.E., Lynas-Gray A.E., Smith G., 1995, A\&A 296, 217

Carney B.W., 1979, ApJ 233, 211

Carney B.W., Latham D.W., Laird J.B., Aguilar L.A., 1994, AJ 107,2240

Cayrel de Strobel G., Hauck B., Francois P., et al., 1992, A\&AS 95, 273

Cohen M., Walker R.G., Barlow M.J., Deacon J.R., 1992, AJ 104,1650

Edvardsson B., Andersen J., Gustafsson B., et al., 1993, A\&A 275,101

ESA, 1997, The Hipparcos Catalogue, ESA SP-1200

Fuhrmann K., Axer M., Gehren T., 1994, A\&A 285, 585

Glushneva I.N., Voloshina I.B., Knyazeva L.N., 1993, Inside the Stars, IAU Colloquium 137, ASP Conf. Ser. 40, 1993, Weiss W.W. \& Baglin A. (eds.)

Gustafsson B., Bell R.A., Eriksson K., Nordlund Å., 1975, A\&A 42,407

Hauck B., 1994, Intrinsic Colours of MK Types in the Geneva Photometry System. The MK Process at 50 years, ASP Conf. Ser. 60, Corbally C.J., Gray R.O. \& Garrison R.F. (eds.)

Heck A., 1977, A\&AS 27, 47

Holweger H., Bard A., Kock M., Kock A., 1991, A\&A 249, 545

Holweger H., Kock M., Bard A., 1995, A\&A 296, 233

Johnson H.L., Mitchell R.I., Iriarte B., Wisniewski W.Z., 1966, Lun. Plan. Lab. Comm. 4, No. 63, 99

Kurucz R.L., 1991, Stellar Atmospheres: Beyond Classical Models, NATO ASI Ser. 341, Crivellari L., Hubeny I. and Hummer D.G. (eds.), p. 441

Kurucz R.L., 1992, The Stellar Population of Galaxies, I.A.U. Sym. 149, Barbuy B. and Renzini A. (eds.). Kluwer, Dordrecht, p. 225

Mégessier C., 1994, A\&A 289, 202

Olsen E.H., 1977, A\&AS 29, 313

Olsen E.H., 1983, A\&AS 54, 55

Olsen E.H., 1993, A\&AS 102, 89

Peterson R.C., Carney B.W., 1979, ApJ 231, 762

Rufener F., 1988, Catalogue of Stars measured in the Geneva Observatory Photometric system (fourth edition). Observatoire de Genève - CH-1290, Sauverny-Switzerland

Saxner M., Hammarbäck G., 1985, A\&A 151, 372

Schuster W.J., Nissen P.E., 1989, A\&A 221, 65

Smalley B., 1993, MNRAS 265, 1035

van der Bliek N.S., Gustafsson B., Eriksson K., 1996a, A\&A 309,849

van der Bliek N.S., Manfroid J., Bouchet P., 1996b, A\&AS 119, 547

van der Bliek N.S., Bouchet P., Habing H.J., et al., 1992, Standard Stars for the Infrared Space Observatory ISO, The Messenger 70, 28

Walker R.G., Cohen M., 1992, An Atlas of Selected Calibrated Stellar Spectra, NASA Contractor Report 17776044, Sept. 1992, p. 144 